\title{
Risk Factors for SARS-CoV-2 Infection Severity in Abu Dhabi
}

\author{
Latifa Mohammad Baynouna AlKetbi ${ }^{1}$ (1) $\cdot$ Nico Nagelkerke ${ }^{2} \cdot$ Hanan Abdelbaqi ${ }^{1} \cdot$ Fatima ALBlooshi $^{1}$. \\ Mariam AISaedi ${ }^{1}$. Shamsa Almansoori ${ }^{1}$ - Ruqaya AINuaimi ${ }^{1}$ - Amal AlKhoori ${ }^{1}$ - Aysha AIAryani ${ }^{1}$ - Mariam AIShamsi ${ }^{1}$. \\ Fatima Kayani ${ }^{1}$. Noura Alblooshi ${ }^{1}$. Shamma AlKhajeh ${ }^{1}$. Jehan AIFalahi ${ }^{1}$. Sumaya AIAmeri ${ }^{3}$. Saeed AIDhahei ${ }^{4}$
}

Received: 19 December 2020 / Accepted: 6 August 2021 / Published online: 23 August 2021

(c) The Author(s) 2021

\begin{abstract}
Background Prediction models are essential for informing screening, assessing prognosis, and examining options for treatment. This study aimed to assess the risk of SARS-CoV-2 infection severity in the Abu Dhabi population.

Methods This is a mixed retrospective cohort study and case-control study to explore the associated factors of receiving treatment in the community, being hospitalized, or requiring complex hospital care among patients with a diagnosis of SARS-CoV-2. Of 641 patients included, 266 were hospitalized; 135 were hospitalized and either died or required complex care, i.e., required ICU admission, intubation, or oxygen and 131 did not develop severe disease requiring complex care. The third group ("controls") were 375 patients who were not hospitalized. Logistic regression analyses were used to study predictors of disease severity.

Results Among hospitalized patients older age and low oxygen saturation at admission were the consistent and strongest predictors of an adverse outcome. Risk factors for the death in addition to age and low oxygen saturation were elevated white blood count and low reported physical activity. Chronic kidney disease and diabetes were also associated with more severe disease in logistic regression. The mortality rate among those with less than 30 min per week of physical activity was $4.9 \%$, while the mortality rate was $0.35 \%$ for those with physical activity $>30 \mathrm{~min}$ at least once a week. The interval from the onset of symptoms to admission and mortality was found to have a significant inverse relationship, with worse survival for shorter intervals.

Conclusion Oxygen saturation is an important measure that should be introduced at screening sites and used in the risk assessment of patients with SARS-CoV-2. In addition, an older age was a consistent factor in all adverse outcomes, and other factors, such as low physical activity, elevated WBC, CKD, and DM, were also identified as risk factors.
\end{abstract}

Keywords SARS-COV-2 $\cdot$ COVID-19 $\cdot$ Disease severity $\cdot$ Hospitalization $\cdot$ Public health $\cdot$ Risk factors

\section{Introduction}

Latifa Mohammad Baynouna AlKetbi

Latifa.mohammad@gmail.com

1 Abu Dhabi Healthcare Services, 81815 Alain, United Arab Emirates

2 UAEU, Community Medicine Department, Alain, United Arab Emirates

3 Abu Dhabi Department of Health, Abu Dhabi, United Arab Emirates

4 Abu Dhabi University, Public Health College, Alain, United Arab Emirates
New pandemics such as COVID-19 caused by SARSCoV-2 often take the world by surprise, and their impact and duration can be difficult to predict. Scientific research works hand-in-hand with public health measures to contain the spread of infections and mitigate their impact. In addition to basic epidemiology, statistical prediction models are essential for informing screening, exploring prognostic factors, establishing transmission patterns, and examining options for treatment. Models; thus, have been rapidly developed and tested to aid decision-making at points of care [1-3]. A recent meta-analysis showed that the most frequently reported predictors of the prognosis of SARS-CoV-2 
infection are age, body temperature, lymphocyte count, and lung imaging features [2].

The Abu Dhabi-United Arab Emirates represent an excellent setting for this type of data management and analysis, as the healthcare providers responsible for caring for patients with SARS-COV-2/COVID-19 belong to the government hospitals and/or the Ambulatory Healthcare Service, all of which are managed by Abu Dhabi healthcare Services, SEHA. All providers use the same electronic medical record (EMR) system and have received extensive support during the pandemic to provide care for all positive cases as needed [4]. This study aimed to assess the risk of severe COVID-19 disease in the Abu Dhabi population.

\section{Methods}

The study design was a retrospective case-control study. Cases were SARS-Cov-2 patients who required either simple hospitalization or hospitalization with more complex care while the controls were SARS-Cov-2 patients who were not hospitalized. Patients were thus stratified into three groups: (i) patients who were hospitalized and required complex care, such as required oxygenation, ICU admission, or intubation or died, (ii) patients who were hospitalized, but did not require complex care, and (iii) "control" patients who tested positive, but were not hospitalized (followed in the community).

The data sources were Electronic Medical Records reports. Cases were randomly selected patients from all Abu Dhabi hospitals admission database that included all patients admitted to any of Abu Dhabi's SARS-CoV-2-dedicated hospitals. Control patients were randomly selected patients from the centralized data base patients attending the widely distributed screening centers established by Governmental Ambulatory Healthcare Services who tested positive for SARS-CoV-2 by PCR. The majority of these patients did not meet the UAE hospital admission criteria published by the Ministry of Health [5], and were either followedup in the community in specially built institutions or hotels for isolation. All patients, both from hospitals (cases) and screening centers (control), were contacted by telephone for a standardized interview to collect additional data. All subjects were recruited from the two cities of Abu Dhabi Emirate, viz. Abu Dhabi City, and Al Ain between March 1st 2020 to the end of June 2020.

One thousand subjects from these two Abu Dhabi Emirates SARS-CoV-2 databases were randomly selected; 500 from SARS-CoV-2 screening centers database and 500 SARS-CoV-2 admitted patients. After exclusion of 187 nonrespondents for the interview call and 172 subjects with incomplete or inconsistent EMR data (Fig. 1) 641
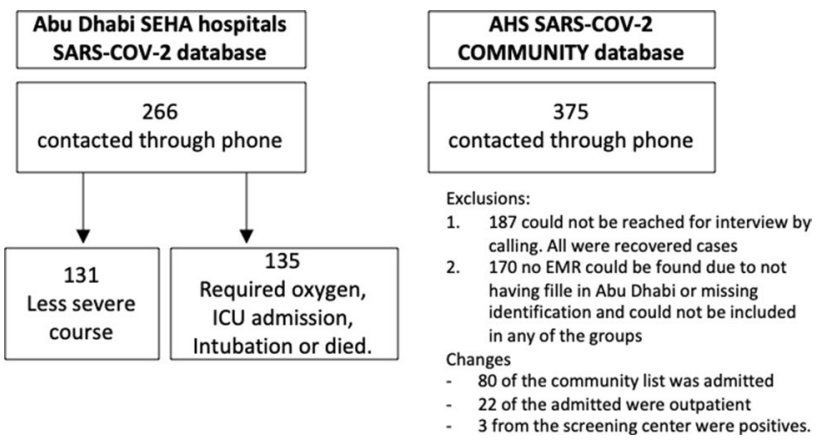

Fig. 1 Subjects numbers and sources included in the different severity cohorts in the study

were included in total. Eighty patients from the community list (i.e., screening centers) were also subsequently admitted to hospital, so included patients were 375 from the screening centers database who were never admitted to hospital and 266 from the admitted list (186 randomly selected from the admitted list plus 80 from the community list who were also admitted). Those who did not respond to the calls had a similar gender distribution and average age as those who responded (64\% male and 36\% females, 35 years mean age for both groups), but they were more likely to be non-UAE nationals ( $83 \%$ vs $16.5 \%$ were non-UAE nationals).

The data were collected by two methods; the data retrieved from the EMR and data collected through a standardized telephone interview conducted in Arabic and English languages as needed. The interview included demographic questions, such as age, gender, nationality, marital status, place of residence, education, and occupation; questions related to their presenting symptoms, if any, and comorbidities; and questions about health-related lifestyle habits, such as smoking, physical activities, and diet. As well, social habits of gatherings and work and travel were included. A copy of the questionnaire used for the interview is included as Supplementary Material.

The EMRs of patients were reviewed manually to ensure accuracy of data collection, consistency of the timeline of events, and correct interpretation of free text variables. The following information was collected: presenting symptoms (if any), contact history, travel history, start of first symptom, temperature, blood pressure, pulse, respiratory rate, oxygen saturation, weight, BMI, CBC ( $\mathrm{Hb}$, Hct, platelets, MCV, MCH), hemoglobinopathies if available, LFT (ALT, AST, AP, GGT), renal function (K, Na, eGFR), vitamin D, coagulation profile (INR, PT, PTT, D dimer), LFT, viral studies, and comorbid conditions. In addition, the outcome of infection and treatments received were collected. There were many missing values for patients who only received 
Table 1 Characteristics of the subjects in each group

\begin{tabular}{|c|c|c|c|c|}
\hline & \multicolumn{4}{|l|}{ SARS-COV-2 } \\
\hline & $\begin{array}{l}\text { Total of all } \\
\text { SARS-COV-2 }\end{array}$ & Community & $\begin{array}{l}\text { Admitted with no } \\
\text { severe illness }\end{array}$ & $\begin{array}{l}\text { Admitted } \\
\text { with severe } \\
\text { illness }\end{array}$ \\
\hline \multicolumn{5}{|l|}{ Age } \\
\hline$<=30$ & $234(36.5)$ & $149(39.7 \%)$ & $52(39.7 \%)$ & $33(24.4 \%)$ \\
\hline $31-40$ & $184(28.7)$ & $138(36.8 \%)$ & $26(19.8 \%)$ & $20(14.8 \%)$ \\
\hline $41-50$ & $113(17.6)$ & $58(15.5 \%)$ & $28(21.4 \%)$ & $27(20 \%)$ \\
\hline $51-60$ & $55(8.6)$ & $24(6.4 \%)$ & $12(9.2 \%)$ & $19(14.1 \%)$ \\
\hline $61-70$ & $28(4.4)$ & $4(1.1 \%)$ & $8(6.1 \%)$ & $16(11.9 \%)$ \\
\hline$>70$ & $27(4.2)$ & $2(0.5 \%)$ & $5(3.8 \%)$ & $20(14.8 \%)$ \\
\hline \multicolumn{5}{|l|}{ Gender } \\
\hline Female & $202(31.5 \%)$ & $114(30.4 \%)$ & $45(34.4 \%)$ & $43(31.9 \%)$ \\
\hline Male & $439(68.4 \%)$ & $261(69.6 \%)$ & $86(65.6 \%)$ & $92(68.1 \%)$ \\
\hline \multicolumn{5}{|l|}{ Marital status } \\
\hline Single & $197(30.7)$ & $121(33.4 \%)$ & $44(33.6 \%)$ & $32(24.1 \%)$ \\
\hline Married & $417(65.1)$ & $240(66.3 \%)$ & $84(64.1 \%)$ & $93(69.9 \%)$ \\
\hline Divorced & $2(0.3)$ & $1(0.3 \%)$ & $1(0.8 \%)$ & $0(0 \%)$ \\
\hline Widowed & $10(1.6)$ & $0(0 \%)$ & $2(1.5 \%)$ & $8(6 \%)$ \\
\hline \multicolumn{5}{|l|}{ Nationality } \\
\hline Non-UAE & 479 (74.7) & $287(77.8 \%)$ & $105(80.2 \%)$ & $87(64.4 \%)$ \\
\hline UAE & $156(24.3)$ & $82(22.2 \%)$ & $26(19.8 \%)$ & $48(35.6 \%)$ \\
\hline \multicolumn{5}{|l|}{ Education } \\
\hline less than high school & $192(30)$ & $94(25.8 \%)$ & $44(34.9 \%)$ & $54(40 \%)$ \\
\hline High School & $211(32.9)$ & $122(33.4 \%)$ & $47(37.3 \%)$ & $42(31.1 \%)$ \\
\hline University or more & $223(34.8)$ & $149(40.8 \%)$ & $35(27.8 \%)$ & $39(28.9 \%)$ \\
\hline \multicolumn{5}{|l|}{ Employment } \\
\hline Employed & 427 (66.6) & $275(73.3 \%)$ & $80(61.1 \%)$ & $72(53.3 \%)$ \\
\hline Unemployed & $37(5.8)$ & $20(5.3 \%)$ & $6(4.6 \%)$ & $11(8.1 \%)$ \\
\hline Housewife & $75(11.7)$ & $25(6.7 \%)$ & $24(18.3 \%)$ & $26(19.3 \%)$ \\
\hline Retired & $17(2.7)$ & $1(0.3 \%)$ & $4(3.1 \%)$ & $12(8.9 \%)$ \\
\hline Student & $40(6.2)$ & $30(8 \%)$ & $4(3.1 \%)$ & $6(4.4 \%)$ \\
\hline Unskilled & $24(3.7)$ & $8(2.1 \%)$ & $11(8.4 \%)$ & $5(3.7 \%)$ \\
\hline \multicolumn{5}{|l|}{ Co-morbidities } \\
\hline $\mathrm{DM}$ & $75(11.7)$ & $27(7.2 \%)$ & $12(9.2 \%)$ & $36(26.7 \%)$ \\
\hline Asthma & $23(3.5)$ & $11(2.9 \%)$ & $2(1.5 \%)$ & $10(7.4 \%)$ \\
\hline Smoker & $57(8.8)$ & $46(12.3 \%)$ & $5(3.8 \%)$ & $6(4.4 \%)$ \\
\hline No chronic illness & $163(25.4)$ & $75(20 \%)$ & $25(19.1 \%)$ & $63(46.7 \%)$ \\
\hline CKD & $19(3)$ & $1(0.3 \%)$ & $6(4.6 \%)$ & $12(8.9 \%)$ \\
\hline Hypertension & $149(23.2)$ & $51(13.6 \%)$ & $41(31.3 \%)$ & $57(42.2 \%)$ \\
\hline \multicolumn{5}{|l|}{ Symptoms } \\
\hline Asymptomatic & $289(45.1)$ & $100(26.7 \%)$ & $95(72.5 \%)$ & $94(69.6 \%)$ \\
\hline Symptomatic & $352(54.9)$ & $275(73.3 \%)$ & $36(27.5 \%)$ & $41(30.4 \%)$ \\
\hline History of cough & $227(35.4)$ & $97(25.9 \%)$ & $70(53.4 \%)$ & $66(48.9 \%)$ \\
\hline History of fever & 267 (41.7) & $157(41.9 \%)$ & $61(46.6 \%)$ & $49(36.3 \%)$ \\
\hline CXR or CT done & $357(55.7)$ & $98(26.1 \%)$ & $126(96.2 \%)$ & $133(98.5 \%)$ \\
\hline \multicolumn{5}{|l|}{ Admissions } \\
\hline Hospitalization & $266(41.5)$ & $0(0 \%)$ & $131(100 \%)$ & $135(100 \%)$ \\
\hline ICU admission & $31(4.8)$ & $0(0 \%)$ & $0(0 \%)$ & $31(23 \%)$ \\
\hline Intubation & $19(3)$ & $0(0 \%)$ & $0(0 \%)$ & $19(14.1 \%)$ \\
\hline Required oxygen & $53(8.3)$ & $0(0 \%)$ & $0(0 \%)$ & $53(39.6 \%)$ \\
\hline Physical activity (tim & & & & \\
\hline
\end{tabular}


Table 1 (continued)

\begin{tabular}{lllll}
\hline & \multicolumn{2}{l}{ SARS-COV-2 } & & \\
\cline { 2 - 5 } & $\begin{array}{l}\text { Total of all } \\
\text { SARS-COV-2 }\end{array}$ & Community & $\begin{array}{l}\text { Admitted with no } \\
\text { severe illness }\end{array}$ & $\begin{array}{l}\text { Admitted } \\
\text { with severe } \\
\text { illness }\end{array}$ \\
\hline$<1$ per week & & & $27(20.6 \%)$ & $31(23 \%)$ \\
$1-2$ per week & $153(23.9)$ & $95(25.9 \%)$ & $48(36.6 \%)$ & $47(34.8 \%)$ \\
$3-4$ per week & $166(25.9)$ & $71(19.3 \%)$ & $34(26 \%)$ & $26(19.3 \%)$ \\
$5-7$ per week & $151(23.6)$ & $91(24.8 \%)$ & $30(22.2 \%)$ \\
$>7$ per week & $155(24.2)$ & $104(28.3 \%)$ & $21(16 \%)$ & $1(0.7 \%)$ \\
Contact history of SARS-COV-2 & $8(1.2)$ & $6(1.6 \%)$ & $1(0.8 \%)$ & $62(47 \%)$ \\
Never & $337(52.6)$ & $212(56.7 \%)$ & $63(48.5 \%)$ & $56(42.4 \%)$ \\
Once & $181(28.2)$ & $66(17.6 \%)$ & $59(45.4 \%)$ & $8(6.1 \%)$ \\
Twice & $30(4.7)$ & $19(5.1 \%)$ & $3(2.3 \%)$ & $1(0.8 \%)$ \\
Three times & $11(1.7)$ & $8(2.1 \%)$ & $2(1.5 \%)$ & $5(3.8 \%)$ \\
More than 3 times & $77(12)$ & $69(18.4 \%)$ & $3(2.3 \%)$ & $10(7.4 \%)$ \\
Travel & $49(7.6)$ & $25(6.7 \%)$ & $14(10.7 \%)$ & 135 \\
Total & 641 & 375 & 131 & \\
\hline
\end{tabular}

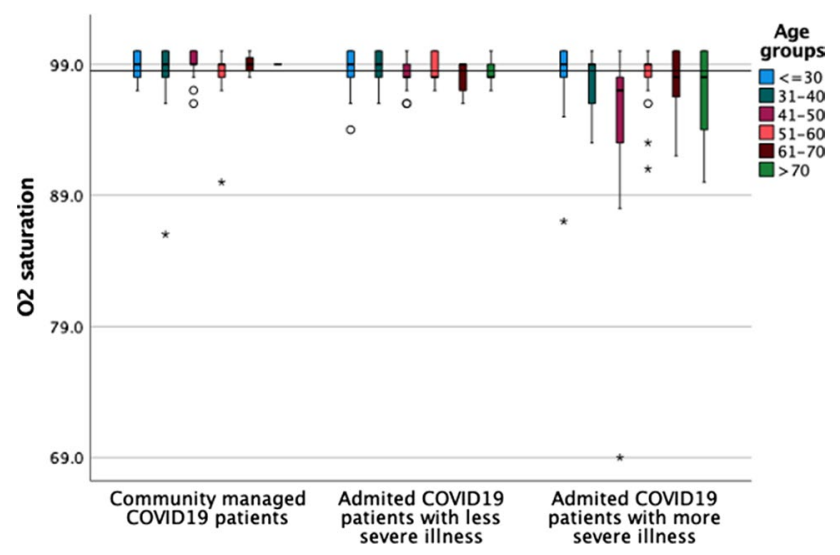

Fig. 2 Oxygen saturation among different severity SARS-COV-2 patients

care in outpatient settings. For deceased patients (9), their close relatives completed the interview questions. No imputation used for the missing data.

Statistical Methods. Sample sizes was calculated on the basis of univariate comparison with $80 \%$ power and $5 \%$ significance level of a dichotomous variable, with prevalences of $30 \%$ and $50 \%$ respectively, between two groups. This yielded a minimum size of 104 patients per group. However, to allow for the presence of three groups as well as multivariate comparisons we decided to expand the sample size to the numbers we could logistically manage. The numbers we obtained were considered adequate in light of the "rule of thumb", often criticized, that the smallest group in a logistic regression analysis should be at least 10 times the number of covariates [6].

Statistical analysis was done using SPSS version 27. Frequencies, cross tabulation in addition to logistic and ordinal regression and survival analysis. Ordinal regression was used to study severity levels reflected in either receiving treatment in the community, being hospitalized or requiring complex care in the hospital among patients with a diagnosis of SARS-CoV-2. Logistic regression (stepwise) was used to study the binary outcomes as mortality, intubation, hospitalization, and ICU admission. Kaplan-Meier curves and log rank test were used for survival analysis. All possible influencers from the variables collected were tested in the mentioned analysis. Complete case analysis was used in handling missing values. The potential impact of the missing data and their exclusion is not expected to cause systemic bias because we can expect missing data are missing completely at random (MCAR) within each severity group. With few exceptions, such as coagulation profiles in nonhospitalized patients no variable was missing more than $40 \%$. In fact, most missingness levels were far lower. 
Table 2 Risk factors of hospitalization, complex care requirement, and adverse outcome among patients with SARS-COV-2 in Abu Dhabi

\begin{tabular}{|c|c|c|c|c|c|c|}
\hline & Estimate & S.E & $P$ value & \multicolumn{2}{|c|}{ Lower bound } & Upper bound \\
\hline \multicolumn{7}{|c|}{$\begin{array}{l}\text { Predictors of hospitalization and complex care } \\
\text { requirement from Ordinal regression }\end{array}$} \\
\hline Age & 0.022 & 0.006 & $<0.001$ & 0.011 & & 0.33 \\
\hline $\mathrm{O}_{2}$ saturation & -0.29 & 0.057 & $<0.001$ & -0.40 & & -0.18 \\
\hline Presenting with symptoms & 1.35 & 0.203 & $<0.001$ & 0.95 & & 1.75 \\
\hline \multirow[t]{2}{*}{ Diabetes mellitus } & 0.82 & 0.303 & 0.007 & 0.22 & & 1.41 \\
\hline & $B$ & S.E & $P$ value & $\operatorname{Exp}(B)$ & \multicolumn{2}{|l|}{$\begin{array}{l}\text { 95\% C.I. for } \\
\text { EXP(B) }\end{array}$} \\
\hline \multicolumn{7}{|c|}{ Predictors of mortality from logistic regression } \\
\hline Age & 0.257 & 0.107 & 0.016 & 1.293 & 1.05 & 1.593 \\
\hline $\mathrm{O}_{2}$ saturation & -0.36 & 0.178 & 0.043 & 0.698 & 0.492 & 0.989 \\
\hline WBC & 0.496 & 0.221 & 0.025 & 1.642 & 1.066 & 2.531 \\
\hline History of increased Physical activity & -4.215 & 1.732 & 0.015 & 0.015 & 0 & 0.44 \\
\hline \multicolumn{7}{|c|}{ Predictors of ICU admission from logistic regression } \\
\hline Age & 0.068 & 0.016 & 0 & 1.07 & 1.037 & 1.105 \\
\hline $\mathrm{O}_{2}$ saturation & -0.378 & 0.076 & 0 & 0.685 & 0.59 & 0.796 \\
\hline WBC & 0.177 & 0.072 & 0.014 & 1.194 & 1.037 & 1.375 \\
\hline CKD & 1.277 & 0.646 & 0.048 & 3.585 & 1.01 & 12.727 \\
\hline \multicolumn{7}{|c|}{ Predictors of intubation from logistic regression } \\
\hline Age & 0.064 & 0.018 & 0 & 1.066 & 1.029 & 1.104 \\
\hline $\mathrm{O}_{2}$ saturation & -0.182 & 0.095 & 0.056 & 0.833 & 0.691 & 1.005 \\
\hline WBC & 0.16 & 0.077 & 0.039 & 1.173 & 1.008 & 1.365 \\
\hline
\end{tabular}

\section{Results}

Table 1 shows the number of subjects in each group. There were 641 SARS-CoV-2 cases included in the analysis: 135 admitted and required complex care, 131 admitted with no requirement of complex care and 375 community (not hospitalized) cases. All subjects had tested positive for SARS-CoV-2-by PCR with the exception of 10 subjects with a negative test but who were diagnosed clinically, e.g., on the basis of CT findings. The mean ages among the different severity groups of patients diagnosed with SARS-CoV-2 differed markedly. The mean age was 44 years (range: 4 months-76 years) for the severe SARSCoV-2 patients, 35 years ( 4 months-75 years) for those who were admitted, but not in the severe category, and 32 years (4 months-73 years) for those who were never admitted (Table 1). By logistic regression among all patients, older age, and low oxygen (Fig. 2) saturation at admission were the consistent and strongest predictors of adverse outcomes, including death, ICU admission, intubation, or requirement of oxygen $(P=0.004$ and $P<0.001)$. Other risk factors for severe illness were being diabetic $(B=0.554, P=0.001)$ and presenting with symptoms $(B=0.489, P<0.0001)$ (Table 2).

Out of a severe course of disease, death $(n=9)$ is clearly the worst. The risk of mortality, in logistic regression, among all patients increased with age (OR per year $=1.2, P=0.016)$, low oxygen saturation (OR per $1 \%$ decrease $=0.698, P=0.043)$, elevated white blood count $(\mathrm{OR}=1.64$ per $1 \times 109 / \mathrm{L}$ increase, $P=0.025)$, and low reported physical activity $(\mathrm{OR}=0.015, P=0.015)$. The mortality rate among those with less than 30 min per week of physical activity was $5.04 \%$ as compared to a rate of $0.35 \%$ for those with physical activity $>30 \mathrm{~min}$ at least once per week.

Another adverse development is ICU admission. Only CKD (Chronic Kidney Disease) was found to be associated with an elevated probability of ICU admission, However, 


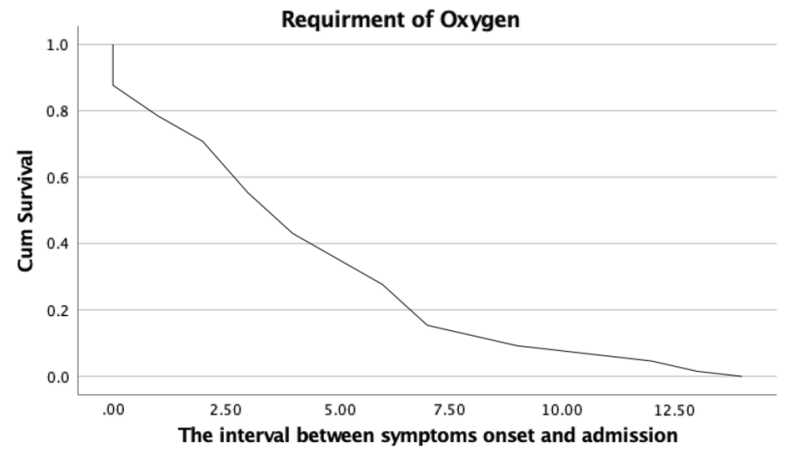

A Kaplan-Meier analysis of the relation between the interval of days between onset of symptoms and admission and the requirement of Oxygen

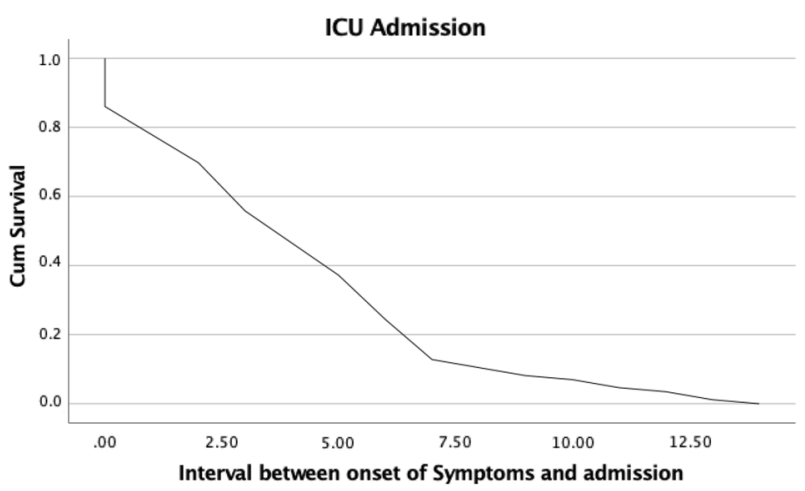

C Kaplan-Meier analysis of the relation between the interval of days between onset of symptoms and admission and the requirement of ICU admission.

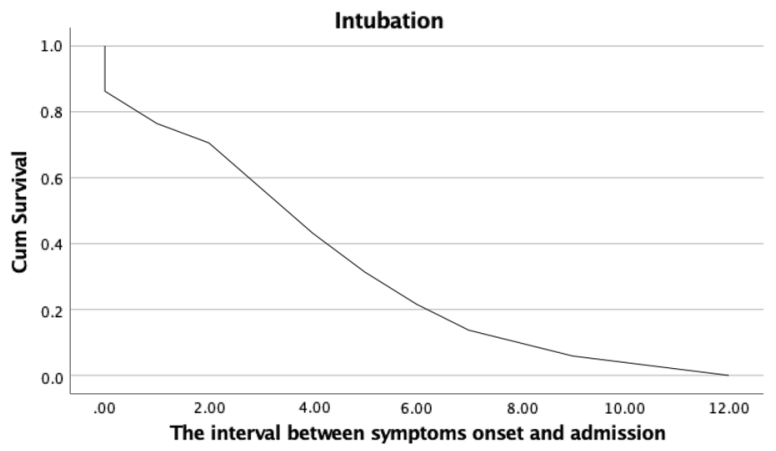

B Kaplan-Meier analysis of the relation between the interval of days between onset of symptoms and admission and the requirement of Intubation.

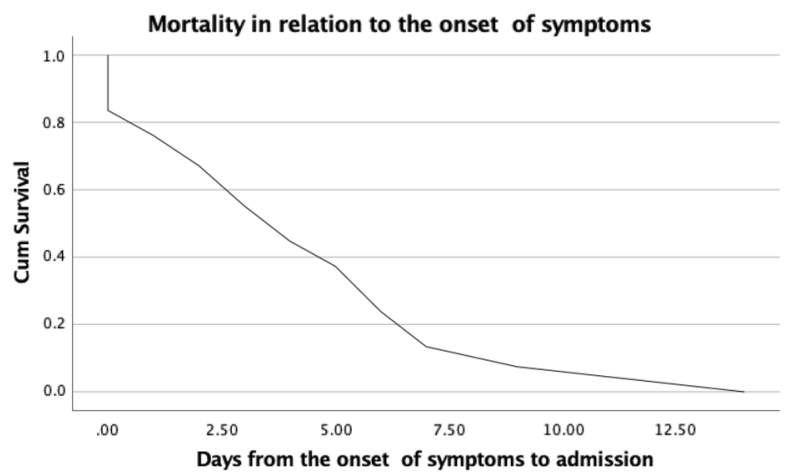

D Kaplan-Meier analysis of the relation between the interval of days between onset of symptoms and admission and Death.

Fig. 3 Kaplan-Meier analysis of the relation between the interval of days between the onset of symptoms among SARS-Cov-2 patients and studied outcomes

our relatively modest sample size could have caused the absence of major chronic illness as risk factor in this model.

Another factor that was studied, by survival analysis, was the relationship between the interval from the onset of symptoms to admission and mortality, which yielded a significant inverse relationship, with worse survival for shorter intervals (Fig. 3).

By ordinal regression, comparing the three groups; not admitted, hospitalized, and hospitalized with complex care requirement, progress in these three levels was only determined by an older age, a lower oxygen saturation (OR (per year $)=0.02, P<0.0001 ;$ OR $($ per $1 \%)=0.29, P<0.0001$, respectively) and being symptomatic(OR $=1.3, P<0.0001)$.
Most of the community treated cohort (73.3\%) were asymptomatic as compared to $27.5 \%$ and $30.4 \%$ ) for the admitted patients' groups. Notably, fever was a strong determinant of admission overall $(\mathrm{OR}=4.3, P<0.0001$ in multivariate logistic regression.

\section{Discussions}

The strongest risk factors for an adverse outcome or severe course of disease among hospitalized patients were advanced age, decreased oxygen saturation, and being symptomatic such as having fever, while elevated white blood count, diabetes, and chronic kidney disease were 
related to some of the adverse outcomes, such as death, ICU admission and intubation. These factors were similarly identified in a systematic review by Wynanuts and colleagues [2] and other studies [3, 7-9]. The present study, however, did not find a significant association between adverse outcomes and the other factors identified in other studies, such as sex, hypertension, respiratory disease, imaging findings, or BMI. However, this reflects the current literature which reports variability in prediction of outcome. Similar to this study, a recent review found no scientific support for claiming that hypertension contribute to unfavorable outcomes in COVID-19 [10]. Another study by Gebhard et al. [11] found sex as a determinant factor of survival. The variability could be attributed in this case to impact of gender-specific lifestyle, health behavior, psychological stress, and socioeconomic conditions difference between different countries. With regards to diabetes mellites Singh et al. [12] found particularly poorly controlled group to be associated with a significantly higher risk of severe COVID-19 and mortality. In this study only a diagnosis of diabetes was among the studied variables and not the glycemic control. Many other factors are being identified [13] and their expression in different setting will be of an interest.

The identification of these factors is important because they can be used, at least locally, to stratify patients and facilitate the management of patients with SARS-COV-2 in addition to adding to the gathered data internationally. Referral protocols will benefit from these findings, especially regarding stratifying the population based on the age and comorbidities and including oxygen saturation when monitoring positive cases. Especially that symptoms were more in groups with less severity. High-risk patients with SARS-CoV-2 as well seem to develop symptoms more rapidly and have a shorter interval from the development of symptoms to admission than low-risk patients, which demonstrates the variation in disease presentation and the need for well-designed prospective studies to determine the underlying etiology.

These factors may change rapidly, and different susceptible groups may emerge [14]; therefore, developing and updating such models are important for maintaining their relevance to efforts to contain the pandemic. For this model to be useful, it is necessary to conduct both regular surveillance and regular data gathering, preferably at the individual level. Risk factors identified in this study could be helpful in identifying patients at risk of severe disease much earlier than on the basis of rapidly worsening symptoms, as is often the case. A case in point is physical activity, with low physical activity a clear predictor of poor outcome.

The strength of this study is the population severity spectrum. The SARS-Cov-2 infection from any positive result in the community from SRS-Cov-2 screening as compared to all admitted patients, hospitalized, and those who required complex care. This might stimulate further studies that is well needed in the UAE as assessing how much care improvement could have been achieved by using this model vs other models, such as the $4 \mathrm{c}$ for example [1]. Another strength is that the study verified data from EMR through chart review to understand the diseases course and timeline of occurrence of events. Nevertheless, this limited the sample size. With the UAE having one of the lowest mortality rates in the world from SARS-Cov-2 larger sample size is needed to identify predictors of less common outcomes. Similar to other prediction models studies these results will require validation studies which are increasingly developing in different region in the world [15].

\section{Conclusion}

Important measures were identified in this study to guide risk assessment of patients with SARS-CoV-2 at least in the UAE such as oxygen saturation, and shorter interval between onset of symptoms and hospitalization. In addition, an older age was a consistent factor in all adverse outcomes, and other factors, such as low physical activity, elevated WBC, CKD, and DM, were also identified as risk factors.

\section{Appendix}

See Table 3. 
Table 3 Checklist of items to include when reporting a study developing or validating a multivariable prediction model for diagnosis or prognosis*

\begin{tabular}{|c|c|c|c|}
\hline Section/topic & $\begin{array}{l}\text { Item Development } \\
\text { or validation? }\end{array}$ & Checklist item & Page \\
\hline
\end{tabular}

Title and abstract

$\begin{array}{lcc}\text { Title } & 1 & \mathrm{D} ; \mathrm{V} \\ \text { Abstract } & 2 & \mathrm{D} ; \mathrm{V} \\ \begin{array}{lll}\text { Introduction } \\ \text { Background and objectives }\end{array} & 3 \mathrm{a} & \mathrm{D} ; \mathrm{V} \\ & & \\ & 3 \mathrm{~b} & \mathrm{D} ; \mathrm{V}\end{array}$

Methods

Source of data

4a $\quad \mathrm{D} ; \mathrm{V}$

4b $\quad \mathrm{D} ; \mathrm{V}$

Participants

5a $\quad \mathrm{D} ; \mathrm{V}$

$5 b \quad \mathrm{D} ; \mathrm{V}$

$5 \mathrm{c} \quad \mathrm{D} ; \mathrm{V}$

Outcome

6a D; V

$6 b \quad \mathrm{D} ; \mathrm{V}$

Predictors $7 \mathrm{a} \quad \mathrm{D} ; \mathrm{V}$

$7 \mathrm{~b} \quad \mathrm{D} ; \mathrm{V}$

Sample size $8 \quad$ D; V

Missing data 9 D; V

Statistical analysis methods $10 \mathrm{a} \quad \mathrm{D}$

$10 \mathrm{~b} \quad \mathrm{D}$

$10 \mathrm{c} \mathrm{V}$

10d D; V

$10 \mathrm{e} \quad \mathrm{V}$

$\begin{array}{lll}\text { Risk groups } & 11 \quad \mathrm{D} ; \mathrm{V}\end{array}$

Development vs. validation $12 \quad \mathrm{~V}$

Results

Participants 13a D; V

13b D; V

$13 \mathrm{c} \quad \mathrm{V}$

Model development

$14 \mathrm{a} \quad \mathrm{D}$
Identify the study as developing and/or validating a multivariable prediction $\quad 1$ model, the target population, and the outcome to be predicted

Provide a summary of objectives, study design, setting, participants, sample $\quad 1$ size, predictors, outcome, statistical analysis, results, and conclusions

Explain the medical context (including whether diagnostic or prognostic) and 3 rationale for developing or validating the multivariable prediction model, including references to existing models

Specify the objectives, including whether the study describes the development or validation of the model, or both

Describe the study design or source of data (e.g., randomized trial, cohort, or registry data), separately for the development and validation data sets, if applicable

Specify the key study dates, including start of accrual; end of accrual; and, if applicable, end of follow-up

Specify key elements of the study setting (e.g., primary care, secondary care, 4 general population) including number and location of centres

Describe eligibility criteria for participants $\quad 4-5$

Give details of treatments received, if relevant 4

Clearly define the outcome that is predicted by the prediction model, including 7 how and when assessed

Report any actions to blind assessment of the outcome to be predicted NA

Clearly define all predictors used in developing the multivariable prediction $\quad 5$ model, including how and when they were measured

Report any actions to blind assessment of predictors for the outcome and other NA predictors

Explain how the study size was arrived at

5

Describe how missing data were handled (e.g., complete-case analysis, single $\quad 6$ imputation, multiple imputation) with details of any imputation method

Describe how predictors were handled in the analyses

Specify type of model, all model-building procedures (including any predictor selection), and method for internal validation

For validation, describe how the predictions were calculated

NA

Specify all measures used to assess model performance and, if relevant, to compare multiple models

Describe any model updating (e.g., recalibration) arising from the validation, if NA done

Provide details on how risk groups were created, if done

NA

For validation, identify any differences from the development data in setting, NA eligibility criteria, outcome, and predictors

Describe the flow of participants through the study, including the number of participants with and without the outcome and, if applicable, a summary of the follow-up time. A diagram may be helpful

Describe the characteristics of the participants (basic demographics, clinical features, available predictors), including the number of participants with missing data for predictors and outcome

For validation, show a comparison with the development data of the distribution of important variables (demographics, predictors and outcome)

Table 1

Specify the number of participants and outcome events in each analysis

NA

Table 2 
Table 3 (continued)

\begin{tabular}{lcc}
\hline Section/topic & Item $\begin{array}{c}\text { Development } \\
\text { or validation? }\end{array}$ & Checklist item \\
\hline
\end{tabular}

Page 7-8

\begin{tabular}{|c|c|c|c|c|}
\hline & $14 \mathrm{~b}$ & $\mathrm{D}$ & $\begin{array}{l}\text { If done, report the unadjusted association between each candidate predictor and } \\
\text { outcome }\end{array}$ & $7-8$ \\
\hline \multirow[t]{2}{*}{ Model specification } & $15 \mathrm{a}$ & $\mathrm{D}$ & $\begin{array}{l}\text { Present the full prediction model to allow predictions for individuals (i.e., all } \\
\text { regression coefficients, and model intercept or baseline survival at a given } \\
\text { time point) }\end{array}$ & \\
\hline & $15 b$ & $\mathrm{D}$ & Explain how to use the prediction model & $8-9$ \\
\hline Model performance & 16 & $\mathrm{D} ; \mathrm{V}$ & Report performance measures (with CIs) for the prediction model & Table \\
\hline \multicolumn{5}{|l|}{ Page7-8 } \\
\hline Model updating & 17 & $\mathrm{~V}$ & $\begin{array}{l}\text { If done, report the results from any model updating (i.e., model specification, } \\
\text { model performance) }\end{array}$ & NA \\
\hline \multicolumn{5}{|l|}{ Discussion } \\
\hline Limitations & 18 & $\mathrm{D} ; \mathrm{V}$ & $\begin{array}{l}\text { Discuss any limitations of the study (such as nonrepresentative sample, few } \\
\text { events per predictor, missing data) }\end{array}$ & 9 \\
\hline \multirow[t]{2}{*}{ Interpretation } & $19 \mathrm{a}$ & $\mathrm{V}$ & $\begin{array}{l}\text { For validation, discuss the results with reference to performance in the develop- } \\
\text { ment data, and any other validation data }\end{array}$ & NA \\
\hline & $19 \mathrm{~b}$ & $\mathrm{D} ; \mathrm{V}$ & $\begin{array}{l}\text { Give an overall interpretation of the results, considering objectives, limitations, } \\
\text { results from similar studies, and other relevant evidence }\end{array}$ & 9 \\
\hline Implications & 20 & $\mathrm{D} ; \mathrm{V}$ & $\begin{array}{l}\text { Discuss the potential clinical use of the model and implications for future } \\
\text { research }\end{array}$ & 9 \\
\hline
\end{tabular}

Other information

Supplementary information $21 \quad$ D; V

Provide information about the availability of supplementary resources, such as NA study protocol, Web calculator, and data sets

Funding $22 \quad$ D; V

Give the source of funding and the role of the funders for the present study

No funding

*Items relevant only to the development of a prediction model are denoted by $D$, items relating solely to a validation of a prediction model are denoted by $V$, and items relating to both are denoted $D ; V$. We recommend using the TRIPOD Checklist in conjunction with the TRIPOD explanation and elaboration document

From: Transparent reporting of a multivariable prediction model for individual prognosis or diagnosis (TRIPOD): the TRIPOD Statement

Acknowledgements Amna Al Saadi, Mariam Al Kaabi, Muneera Al Blooshi, Raja Farahat, Hodon Saeed, Sameera Omar, Anoud AlShamsi, Mouza Al Dhaheri, Sana Zeinadeen.

Authors contributions All authors approved the manuscript. LBK conceptualization data analysis and writing the manuscript, NN review of data analysis and manuscript. HAB, FAB, MAS, SAM, RAN, AAK, AAA, MAS, FK, NAB, SAK, JAF, participated in data collection, SAD data management, and manuscript review.

\section{Funding None.}

\section{Declarations}

\section{Conflict of interest None.}

Ethics approval and consent to participate This study was approved by the Abu Dhabi COVID19 Research IRB Committee, DOH/ CVDC/2020/833. Written informed consent was obtained from all participants which was at the start of the electronic survey.

Consent for publication Abu Dhabi Health COVID19 Research Ethics Committee consented to publish this study. All authors consented to publish.
Availability of data and materials Available on request based on the Abu Dhabi Health COVID19 Research Ethics Committee approval.

Open Access This article is licensed under a Creative Commons Attribution 4.0 International License, which permits use, sharing, adaptation, distribution and reproduction in any medium or format, as long as you give appropriate credit to the original author(s) and the source, provide a link to the Creative Commons licence, and indicate if changes were made. The images or other third party material in this article are included in the article's Creative Commons licence, unless indicated otherwise in a credit line to the material. If material is not included in the article's Creative Commons licence and your intended use is not permitted by statutory regulation or exceeds the permitted use, you will need to obtain permission directly from the copyright holder. To view a copy of this licence, visit http://creativecommons.org/licenses/by/4.0/.

\section{References}

1. Knight SR, Ho A, Pius R et al. Risk stratification of patients admitted to hospital with covid-19 using the ISARIC WHO clinical characterisation protocol: development and validation of the 4C Mortality Score. BMJ 2020; 370, m3339. 
2. Wynants L, Van Calster B, Collins GS et al. Prediction models for diagnosis and prognosis of covid-19 infection: systematic review and critical appraisal. BMJ 2020; 369, m1328.

3. Zheng Z, Peng F, Xu B, et al. Risk factors of critical \& mortal COVID-19 cases: a systematic literature review and meta-analysis. J Infect. 2020;81:e16-25.

4. Al Falasi RJ, Khan MA. The impact of COVID-19 on Abu Dhabi and its primary care response. Aust J Gen Pract 2020; 49.

5. AUTHORITY DUBAIHEALTH. Guidelines for Healthcare Professionals Managing Covid-19. 2020; Version No. 1.

6. van Smeden M, Moons KG, de Groot JA, et al. Sample size for binary logistic prediction models: beyond events per variable criteria. Stat Methods Med Res. 2019;28:2455-74.

7. Leung C. Risk factors for predicting mortality in elderly patients with COVID-19: a review of clinical data in China. Mech Ageing Dev 2020;188, 111255.

8. Rod JE, Oviedo-Trespalacios O, Cortes-Ramirez J. A briefreview of the risk factors for covid-19 severity. Rev Saude Publica. 2020;54:60.

9. Wolff D, Nee S, Hickey NS, Marschollek M. Risk factors for Covid-19 severity and fatality: a structured literature review. Infection. 2021;49:15-28.
10. Drager LF, Pio-Abreu A, Lopes RD, Bortolotto LA. Is hypertension a real risk factor for poor prognosis in the COVID-19 pandemic. Curr Hypertens Rep. 2020;22:43.

11. Gebhard C, Regitz-Zagrosek V, Neuhauser HK, Morgan R, Klein SL. Impact of sex and gender on COVID-19 outcomes in Europe. Biol Sex Differ. 2020;11:29.

12. Singh AK, Khunti K. Assessment of risk, severity, mortality, glycemic control and antidiabetic agents in patients with diabetes and COVID-19: a narrative review. Diabetes Res Clin Pract $2020 ; 165,108266$.

13. Conca W, Alabdely M, Albaiz F et al. Serum $\beta 2$-microglobulin levels in Coronavirus disease 2019 (Covid-19): another prognosticator of disease severity. PLoS One 2021; 16, e0247758.

14. Sperrin M, McMillan B. Prediction models for covid-19 outcomes. BMJ 2020; 371, m3777.

15. Paiva Proença Lobo Lopes F, Kitamura FC, Prado GF, Kuriki PEA, Garcia MRT, COVID-AI-Brasil. Machine learning model for predicting severity prognosis in patients infected with COVID-19: Study protocol from COVID-AI Brasil. PLoS One $2021 ; 16$, e0245384. 